\title{
If Your Patients Were Too Embarrassed to Go Out in Public, What Would You Do? - Public Education to Break the Stigma on Parkinson's Disease Using Integrated Media
}

This article was published in the following Dove Press journal:

Patient Related Outcome Measures

\section{Priya Jagota $\mathbb{D}^{\prime}$ \\ Porntip Jongsuntisuk' \\ Rachaneewan Plengsri ${ }^{2}$ \\ Marisa Chokpatcharavate ${ }^{2}$ \\ Onanong Phokaewvarangkul' \\ Vittratorn Chirapravati $i^{3}$ \\ Pattamon Panyakaew' \\ Jirada Sringean' \\ Roongroj Bhidayasiri (iD) \\ 'Chulalongkorn Centre of Excellence for Parkinson's Disease and Related \\ Disorders, Department of Medicine, Faculty of Medicine, Chulalongkorn University and King Chulalongkorn Memorial Hospital, Thai Red Cross Society, Bangkok, Thailand; \\ ${ }^{2}$ Chulalongkorn Parkinson's Disease Support Group, Chulalongkorn Centre of Excellence for Parkinson's Disease and Related Disorders, Department of Medicine, Faculty of Medicine, \\ Chulalongkorn University and King Chulalongkorn Memorial Hospital, Tha Red Cross Society, Bangkok, Thailand; ${ }^{3}$ Chulalongkorn University Communication Arts Alumni Association, Faculty of Communication Arts, Chulalongkorn University, Bangkok, Thailand}

Correspondence: Priya Jagota Chulalongkorn Centre of Excellence for Parkinson's Disease and Related

Disorders, Department of Medicine, Faculty of Medicine, Chulalongkorn University and King Chulalongkorn Memorial Hospital, Thai Red Cross Society, 1873 Rama 4 Road, Bangkok 10330, Thailand

Tel $+662256-4000$ ext. 7070 I

Fax +662 256-4630

Email pja@chulapd.org
Purpose: People with Parkinson's (PwP) often feel stigmatized, especially when their symptoms are visually prominent. In some countries, public awareness of Parkinson's disease can be minimal, leading to social challenges for PwP.

Patients and Methods: A public educational campaign using videos (VDO) showing $5 \mathrm{PwP}$ in different social situations that their symptoms could be a source of inconvenience to others were launched on Facebook (FB) and television (TV). We describe the process and report the impact of the campaign on patients and the public. Number of shares and likes and the first 100 comments on FB were analyzed.

Results: There were almost 1 million views, 680,000 likes, and 256,000 shares in the Center's and popular Facebook Pages within 24 hours, subsequently reaching 4.4 million views, 138,000 likes, and 78,000 shares. Patients reported changed behavior and demeanor by the public towards them. The public's comments ranged from stating that they have seen PwP without knowing what it was to requests for more information about PD to thanks for the informative VDO. People also shared their experiences with PwP.

Conclusion: The campaign was impactful in creating awareness about PD. Creating appealing content with appropriate video length and collaborating with community groups that have relevant skills can help in creating and disseminating an educational video which creates a significant impact on society as a whole. This can be emulated to educate the public about other diseases.

Keywords: Parkinson's disease, public education, social media

\section{Introduction}

Parkinson's disease (PD) is a neurodegenerative disease. Nevertheless, people with Parkinson's (PwP) are fully alert and aware of themselves and their surroundings, and with today's medicines, they can be fully mobile and functional. However, there may be periods where they experience "off" symptoms or "expose" some of their symptoms. Our center is a tertiary center for the treatment of Parkinson's disease with an affiliated, fully functional Parkinson's Disease Support Group of over 900 members where PwP and caregivers meet at least 2-3 times/week to do activities. Many PwP had expressed their self-stigma stating social avoidance and being in public because of their disease. One of the main reasons is that when their symptoms are "exposed", they receive a not-so-friendly look from the public as 
a result of causing inconvenience, especially when they are slow and rigid. Most understand that this was due to the public's lack of knowledge and understanding of Parkinson's disease. ${ }^{1}$ Together, we decided to take on an initiative to educate the mass public about PD.

The presence and impact of social media are undeniable. Its reach today is far broader and cheaper than traditional media. Globally, the average time spent on social media is 135 minutes per day. ${ }^{2}$ Facebook (FB) is the most popular social media, with more than 2.2 billion worldwide active users. ${ }^{3}$ The type of posts that get the most engagement are videos (VDOs), especially those with heartwarming and inspiring content. ${ }^{4}$ Globally, Thailand ranks in the top ten countries for social media usage. ${ }^{5}$ A few studies have looked at using social media for the purpose of educating the public on PD. There is a lot of misleading content which has gained popularity. This is partly due to patients' interest in that topic, or the content was more appealing and of a more appropriate length. ${ }^{6,7}$ Through a consensus meeting between the patient's support group, neurologists, and multidisciplinary team at our center (www.chulapd.org), we decided to create an accurate and appealing high-quality VDO to educate the public.

\section{Patients and Methods}

\section{Creating the Media}

We contacted our university's Faculty of Communication Arts to help with this project. The Communication Arts Alumni Association (CAAA) volunteered. There were producers, directors, scriptwriters, and public relations experts, among others. Volunteers from Patients Support Group, our center, and CAAA met a few times to talk about the details of the disease, what makes PwP feel stigmatized and what they want to convey to the public. The CAAA created three storylines for a short film. The first one was "sorry, we have Parkinson's disease". It portrayed $\mathrm{PwP}$ in various day-to-day situations, which can cause inconvenience to others, such as a patient having freezing of gait while crossing the road, a middle-aged woman having marked tremor in an office, and an elderly woman having a masked face while family members talk and laugh. Each scene ended with PwP folding hands and saying, "sorry that we have Parkinson's disease". The second storyline titled "Do I really have to be with this kind of person?" shows similar scenarios portrayed as being "ill-mannered". For example, a "person" stops in the middle of the road while the drivers are waiting, and a "person" does not laugh at a joke while all the others laugh. The third storyline depicts the life of a father and son before discovering that the father had PD. It shows the son being frustrated and angry at Dad all the time when Dad is slow to do things and too "lazy" to button up his shirt. The three storylines were shown to the PwP of the Parkinson's Disease Support Group. The PwP voted and chose the first storyline for the short film. It was discussed to be adapted to show PwP in those situations with a common public reaction. The title "sorry" and the scenes showing PwP folding hands and apologizing were dropped as the group felt that PwP should not be sorry about their disease. The mnemonic "3S-Stiff, Shake, Slow" was used in the VDO with the message "understand patients with Parkinson's". Real PwP were casted and "acted" in the film. All patients and participants signed a consent form permitting dissemination and publication of the VDO. The story was filmed and edited into a 60 seconds VDO for TV channels, one 90 seconds VDO and four 15 seconds VDOs showing each PwP's symptoms in detail for social media. The hashtag \#UnderstandParkinson's in Thai was used. The VDO was highly emotional and the messages were very focused and brief to catch the public's attention.

\section{Disseminating the Media}

Boosting posts on FB and disseminating the VDO in traditional media (together called integrated media) can be very expensive. The CAAA contacted celebrities and advertising media agencies to help show the film free of charge on various media platforms. The VDO clip was launched on our official Facebook Page (Parkinson Chula https://www.facebook.com/114128941932756/ videos/1873937219285244/) on a predetermined date (see Video 1 for VDO with English subtitle). On the next day, celebrities simultaneously posted their pictures in "shaking" mode on Instagram. While this may be seen differently in some cultures, in Thai culture, it showed a symbol of support for the PwP's symptoms. Other VDOs were subsequently released within a few days' gaps. VDOs were also shown on various TV shows, advertising platforms, such as digital billboards, LCDs in buses, trains, department stores and popular Facebook Pages (>1 million followers) (Figure 1).

\section{Measuring the Impact}

After the launch of the campaign, we sought to measure its impact. Ethics approval was granted by Chulalongkorn University, Faculty of Medicine's Institutional Review 
Board (IRB), IRB no. 703/2020. The impact on patients and the public was measured using the following metrics: 1 . Number of views and shares on FB - this would quantitatively measure the reach of the campaign in social media. 2 . Patient's comments on FB and at our support group - this would measure the campaign's direct impact on PwP. 3. Public's comments on FB - this would measure the impact on the public. The first 100 comments on FB were quantitatively analyzed and categorized to study types of comments.

\section{Results}

The results of measuring the impact of the campaign using the described metrics are described below:

1. Campaign's reach in social media: There were almost 1 million views, 680,000 likes, and 256,000 shares in the Center's and popular Facebook Pages within 24 hours. Subsequently, over a few months, the Center's Page alone reached 4.4 million views, 138,000 likes, and 78,000 shares. Also, various TV shows contacted our Center to talk about PD on their shows.

2. Direct impact on PwP: There were no comments by a PwP in the first 100 comments on the FB page. However, there were comments by PwP on how their lives have changed after the campaign in our support group. People were more understanding and less judgmental. They were offered more help in public. To quote, one of them said, "Whenever I used to go to the market when having dyskinesia, people would always think that I was drunk. However, now they understand that it is my Parkinson's". Another patient mentioned, "I used to go to a hair salon. There were young hairdressers there. They would always feel irritated at my slowness. Recently I went to the same salon. The hairdressers were more patient and asked whether I had Parkinson's".

3. Impact on the public: The summary of types of comments on FB is provided in Table 1. Many mentioned that they had seen people with the symptoms before but never knew what it was. Now they know. There were also many comments from friends and family members of PwP, relating their experience with patients and asking for understanding from the public. Some of the comments made by the public are quoted below:

No. 2: There is a cashier in a department store that I often visit, who always gives my change very very slowly and does not show any facial expression, just like in the VDO. I always get annoyed. But, after watching this VDO, I think that maybe she has Parkinson's.

No. 10: I had heard of PD before but never knew the symptoms. I have seen people who have symptoms similar to the ones in the VDO before. I always complain in my mind as they do everything very slowly. Now I understand them more. Thank you for the good VDO.

No. 21: My father has Parkinson's. He is embarrassed by his tremor, so he does not go out of the house.

No. 26: I think my mother might have Parkinson's.

No. 30: My grandmother had Parkinson's. I never realized how much she suffered! Nobody understood her!

No. 32: My father has had Parkinson's for over 20 years. We have to be very patient and understanding.

No. 34: My father has Parkinson's and feels embarrassed that he does things slower than the others. But now he seems better.

No. 60: Should there be a symbol for PwP for the public to recognize those in need of help?

No. 65: I have experience trying to help but was refused. I realized that not everybody wants help from others.

No. 72: Can you please explain the symptoms in more detail? I meet people with similar symptoms off and on. I want to understand them more.

No. 80: I understand the uncle that sells banana sticky rice now.

The total media value reached was approximately US\$ 1.5 million (Table 2).

\section{Discussion}

In a world where the public is continually bombarded by VDOs and pictures, especially in social media, it is crucial to create attractive, meaningful and succinct VDOs to catch the attention of the public. Experts in two fields movement disorders and communication arts, collaborated to create the VDO for this campaign to ensure the accuracy of the information with an appeal to the public. They took into consideration the storyline which was emotional yet inspiring, accuracy of information, quality of production, and length of the VDO. Additionally, PwP were involved in every step of the campaign. They chose the storyline, "acted" in the film, and helped answer questions on social media. 


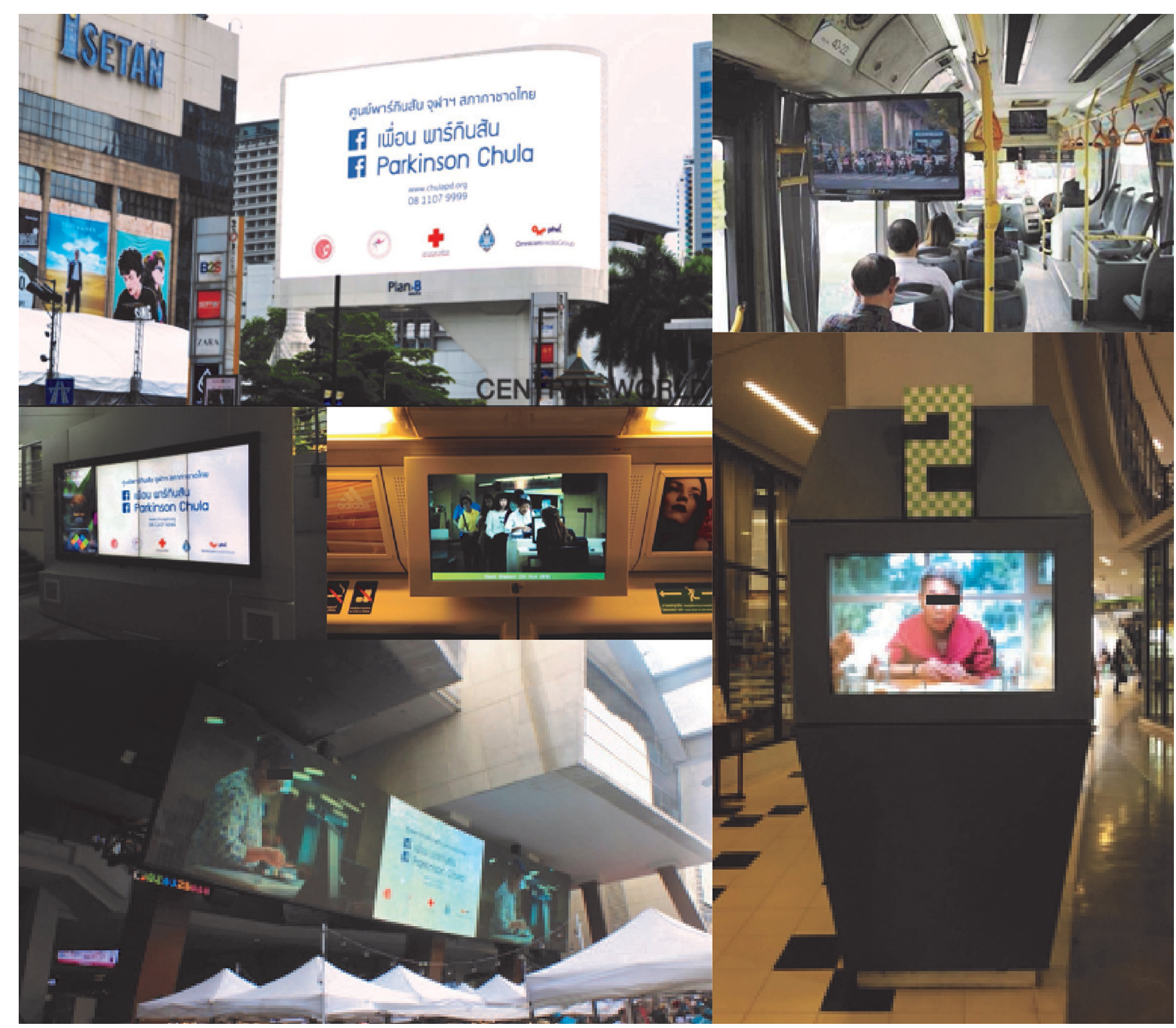

Figure I The picture shows the VDO being shown on digital billboards in front of department stores, on LCDs in buses, trains, and inside department stores.

As this was a social campaign disseminated nationwide and on various platforms, it is difficult to truly quantitatively measure the knowledge gained by the public. Nonetheless, we have attempted to quantify the impact by looking at the number of likes and shares, and analyzing the first 100 comments on the FB page. People's comments on FB and in the media showed an increased awareness and concern for PwP. After the VDO launched on $\mathrm{FB}$, many TV programs (not contacted by the CAAA) contacted the center to talk about PD on their channel. Many programs dedicated one full episode to the discussion. This in itself showed that the media and the public had increased awareness of PD and PwP.

The expenses of the campaign were only for the preparation of the shoot. Apart from that, everything was on a volunteer basis - shooting, producing, acting, and posting on different media platforms by celebrities, various people and agencies. Many of these were possible because of a culture that is unique to Buddhism and Thailand.

Thailand is a Buddhist country. Merit making is one of the foremost teachings in Buddhism and is an integral part of Thai culture. People are always looking for a chance to make merit in the hope of having a good future. Volunteering to help patients using their expertise, posting the VDOs on their respective social media, and giving media space and time free of charge are all, knowingly or unknowingly, a part of this culture. 
Table I Analysis of the First 100 Comments on Facebook

\begin{tabular}{|l|l|}
\hline Types of Comments & $\begin{array}{l}\text { No. of } \\
\text { Comments }\end{array}$ \\
\hline $\begin{array}{l}\text { Have seen a person with Parkinson's before and } \\
\text { feel for them } \\
\text { Have seen someone with similar "behavior" but } \\
\text { never knew what it was }\end{array}$ & 6 \\
$\begin{array}{l}\text { Relating personal experience with a family member, } \\
\text { friend, etc. }\end{array}$ & 28 \\
$\begin{array}{l}\text { Asking whether they or a family member has } \\
\text { Parkinson's }\end{array}$ & 1 \\
$\begin{array}{l}\text { Asking for more information about Parkinson's } \\
\text { Asking to be referred } \\
\text { Suggesting Marijuana } \\
\text { General comments, thanking for the information, } \\
\text { sharing the VDO }\end{array}$ & 39 \\
\hline
\end{tabular}

Table 2 Types of Media That the VDOs Were Broadcasted and Their Monetary Value

\begin{tabular}{|l|l|l|l|}
\hline \multirow{4}{*}{$\begin{array}{l}\text { Mraditional } \\
\text { Media }\end{array}$} & Media & $\begin{array}{l}\text { Value } \\
\text { (US } \$ \text { ) }\end{array}$ & Detail \\
\cline { 2 - 4 } & $\begin{array}{l}\text { Media on transportation } \\
\text { platforms }\end{array}$ & 309,604 & 360 Spots \\
\cline { 2 - 4 } & Digital screens/billboards & 54,040 & $\begin{array}{l}400 \text { Buses } \\
\text { and trains }\end{array}$ \\
\hline \multirow{4}{*}{$\begin{array}{l}\text { Social } \\
\text { Media }\end{array}$} & $\begin{array}{l}\text { FB Page post/Popular FB } \\
\text { Page partners }\end{array}$ & 107,692 & II Pages \\
\cline { 2 - 4 } & $\begin{array}{l}\text { Influencers - Social media } \\
\text { post (IG) }\end{array}$ & 492,308 & 60 People \\
\cline { 2 - 4 } & $\begin{array}{l}\text { Real users - Social media } \\
\text { post (IG) }\end{array}$ & 369,230 & 400 People \\
\cline { 2 - 4 } & Online interviews & 84,615 & 4 Times \\
\hline \multirow{4}{*}{ Other } & Other & 64,615 & \\
\cline { 2 - 5 } & Total value & $1,536,144$ & \\
\hline
\end{tabular}

Abbreviations: FB, Facebook; IG, Instagram.

\section{Conclusion}

Mass public education about PD or any other neurodegenerative diseases can be achieved with minimal budget through creating engaging content and collaborating with community groups with relevant skills and expertise. These can have a significant impact on the public and patients. Many of these can be emulated in other countries and for other diseases.

\section{Acknowledgments}

We would like to thank the Chulalongkorn University Communication Arts Alumni Association, volunteers, PwP, advertising agencies, and caregivers who took part in the campaign for their tireless contribution and continued support.

\section{Funding}

Part of this campaign was supported by the International Research Network grant (IRN59W0005) of the Thailand Research Fund, Chulalongkorn Academic Advancement Fund into its $2^{\text {nd }}$ Century Project of Chulalongkorn University (2300042200) and Center of Excellence grant of Chulalongkorn University (GCE 6100930004-1).

\section{Disclosure}

Dr. Onanong Phokaewvarangkul has Intellectual Property rights for a portable tremor detection and suppression device and has received grants from the Thailand Research Fund and the Neurological Society of Thailand. Dr. Pattamon Panyakaew has received grants from Rachadapiseksomphot Endowment Fund and Chulalongkorn Academic Advancement Fund into Its $2^{\text {nd }}$ Century Project of Chulalongkorn University. Dr. Jirada Sringean has Intellectual Property rights for NIGHT-Recorder ${ }^{\circledR}$ and has received grants from Rachadapiseksomphot Endowment Fund. Dr. Roongroj Bhidayasiri has Intellectual Property rights for Parkinson's laser cane, Tremor diagnostic algorithm, and NIGHT-Recorder ${ }^{\mathbb{R}}$, received honoraria from BoehringerIngelheim, Novartis, BL-Hua, Abbott, Ipsen, and Britannia pharmaceuticals, receives royalties from Wiley-Blackwell and Humana Press and has received grants from the Newton Fund-UK, the International Research Network grant of the Thailand Research Fund, Chulalongkorn Academic Advancement Fund into Its $2^{\text {nd }}$ Century Project of Chulalongkorn University, Center of Excellence grant of Chulalongkorn University, and Grant for International Research Integration, Chula Research Scholar and Rachadapiseksomphot Endowment Fund. The authors report no other potential conflicts of interest for this work.

\section{References}

1. Jitkritsadakul O, Boonrod N, Bhidayasiri R. Knowledge, attitudes and perceptions of Parkinson's disease: a cross-sectional survey of Asian patients. J Neurol Sci. 2017;374:69-74. doi:10.1016/j.jns.2016.12.063

2. Statista. Daily time spent on social networking by internet users worldwide from 2012 to 2017 (in minutes). Statista-The Statistics Portal. 2019. Available from: https://www.statista.com/statistics/433871/ daily-social-media-usage-worldwide. Accessed February 4, 2019. 
3. Statista. Number of monthly active Facebook users worldwide as of 4th quarter 2018 (in millions). Statista-The Statistics Portal .2019. Available from: https://www.statista.com/statistics/264810/number-of -monthly-active-facebook-users-worldwide. Accessed February 4, 2019.

4. Moeller S. The 2019 Ultimate Guide to Facebook Engagement. Buzzsumo. 2019. Available from: https://buzzsumo.com/blog/face book-engagement-guide/?utm_source=newsletter\&utm_medium $=$ email\&utm_campaign=non-european. Accessed February 4, 2019.

5. Leesa-nguansuk S. Thailand tops internet usage charts. Bangkok Post. February 6, 2018; Business.
6. Kim R, Park HY, Kim HJ, Kim A, Jang MH, Jeon B. Dry facts are not always inviting: a content analysis of Korean videos regarding Parkinson's disease on YouTube. J clin neurosci. 2017;46:167-170. doi:10.1016/j.jocn.2017.09.001

7. Al-Busaidi IS, Anderson TJ, Alamri Y. Qualitative analysis of Parkinson's disease information on social media: the case of YouTube. EPMA J. 2017;8(3):273-277. doi:10.1007/s13167-017-0113-7

\section{Publish your work in this journal}

Patient Related Outcome Measures is an international, peer-reviewed, open access journal focusing on treatment outcomes specifically relevant to patients. All aspects of patient care are addressed within the journal and practitioners from all disciplines are invited to submit their work as well as healthcare researchers and patient support groups.

Submit your manuscript here: http://www.dovepress.com/patient-related-outcome-measures-journal
The manuscript management system is completely online and includes a very quick and fair peer-review system. Visit http://www. dovepress.com/testimonials.php to read real quotes from published authors. 